\title{
CAPACITAÇÃO EM CIRURGIA ROBÓTICA NO PROGRAMA DE RESIDÊNCIA EM ENFERMAGEM PERIOPERATÓRIA
}

\author{
Robotic surgery training in the perioperative nursing residency program \\ Capacitación en cirugía robótica en el programa de residencia en enfermería perioperatoria
}

Cristina Silva Sousa', Daniela Magalhaes Bispo ${ }^{2}$, Ana Lucia Mirancos da Cunha ${ }^{3}$

\begin{abstract}
RESUMO: Objetivo: Desenvolver um programa de treinamento para enfermeiros residentes em cirurgia robótica. Método: Trata-se de um relato de experiência em um hospital filantrópico privado de grande porte no município de São Paulo. Para o programa de residência em Enfermagem foi desenvolvido um treinamento para enfermeiras residentes com foco na especialização em cirurgia robótica com carga teórico-prática para o sistema do robô Da Vinci. Resultados: As enfermeiras residentes desenvolveram o conhecimento cognitivo com o treinamento virtual e a habilidade técnica durante a simulação com o manuseio do robô, dos instrumentais e dos equipamentos. Foram liberadas para iniciação prática com supervisão de enfermeiro especialista até que possuam segurança na execução do procedimento de montagem do robô Da Vinci. Conclusão: O treinamento foi avaliado pelas residentes como satisfatório para aquisição de conhecimento teórico-prático. A capacitação de profissionais especialistas em cirurgia robótica é um diferencial na residência de Enfermagem perioperatória. Palavras-chave: Enfermagem perioperatória. Treinamento por simulação. Procedimentos cirúrgicos robóticos. Educação em Enfermagem. Internato não médico.
\end{abstract}

ABSTRACT: Objective: To develop a training program in robotic surgery addressed to resident nurses. Method: This is a case report conducted in a major private, philanthropic hospital in the city of São Paulo. For the Nursing Residency Program, resident nurses were trained focusing on the specialization in robotic surgery, including theory and practice, to use the Da Vinci robot system. Results: The resident nurses developed cognitive knowledge through virtual training, and technical skills during the simulation of robot handling, as well as the instruments and the equipment. They were referred to practical initiation, supervised by an expert nurse, until they were confident to execute the procedure of assembling the Da Vinci robot. Conclusion: The residents considered the training satisfactory to acquire theoretical and practical knowledge. The training of professionals specialized in robotic surgery is a differential in perioperative Nursing residency. Keywords: Perioperative nursing. Simulation training. Robotic surgical procedures. Education, Nursing. Internship, nonmedical.

RESUMEN: Objetivo: Desarrollar un programa de capacitación para enfermeros residentes en cirugía robótica. Método: Se trata de un relato de experiencia en un hospital filantrópico privado de grande porte en el municipio de São Paulo. Para el programa de residencia en Enfermería fue desarrollada una capacitación para enfermeras residentes con enfoque en la especialización en cirugía robótica con carga teórico-práctica para el sistema del robot Da Vinci. Resultados: Las enfermeras residentes desarrollaron el conocimiento cognitivo con la capacitación virtual y la habilidad técnica durante la simulación con el manejo del robot, de los instrumentos y de los equipos. Fueron liberadas para iniciación práctica con supervisión de enfermero especialista hasta que posean seguridad en la ejecución del procedimiento de montaje del robot Da Vinci. Conclusión: La capacitación fue evaluada por las residentes como satisfactorio para adquisición de conocimiento teórico-práctico. La capacitación de profesionales especialistas en cirugía robótica es un diferencial en la residencia de Enfermería perioperatoria.

Palabras clave: Enfermería perioperatoria. Entrenamiento simulado. Procedimientos quirúrgicos robotizados. Educación en Enfermería. Internado no médico.

'Enfermeira. Doutora em Enfermagem pela Escola de Enfermagem da Universidade de São Paulo (EEUSP). Enfermeira do Centro Cirúrgico do Hospital Sírio-Libanês - São Paulo (SP), Brasil. E-mail: crissousa@usp.br Rua Professora Carolina Ribeiro, 20, apartamento 54 - Vila Mariana - CEP 04116-020 - São Paulo (SP), Brasil.

Enfermeira. Especialista em Enfermagem em Centro Cirúrgico. Enfermeira da Unidade Pré-operatória do Hospital Sírio-Libanês - São Paulo (SP), Brasil.

${ }^{3}$ Enfermeira. Mestre em Enfermagem pela Escola de Enfermagem da Universidade de São Paulo (EEUSP). Enfermeira Coordenadora do Centro Cirúrgico do Hospital Sírio-Libanês -São Paulo (SP), Brasil.

Recebido: 27 maio 2016 - Aprovado: 19 out. 2016

DOI: $10.5327 / Z 1414-4425201600040004$ 


\section{INTRODUÇÃO}

A cirurgia robótica ganhou popularidade, com crescimento rápido desde os anos 2000 . O procedimento permite segurança e eficiência, e situa-se entre a laparoscopia e a cirurgia minimamente invasiva ${ }^{1}$. Na literatura, encontram-se descritos procedimentos robóticos nas especialidades de urologia, ginecologia, cirurgia geral, torácica, cardíaca, cabeça e pescoço, maxilofacial e pediátrica ${ }^{1-4}$. Esse avanço tecnológico vem de encontro ao objetivo de diminuir a morbidade e mortalidade operatória, e realizar cirurgias menos agressivas e com recuperação precoce do paciente ${ }^{5}$.

O enfermeiro do século XXI tem como desafio a diversidade tecnológica, que exige aperfeiçoamento para as novas demandas, atualização constante e busca de capacitação para atender diferentes recursos ${ }^{6}$. A robótica deu ao enfermeiro perioperatório a oportunidade de adaptar a sua prática, pensar criativamente e desenvolver práticas clínicas eficazes e seguras para o cuidado de seu paciente ${ }^{7}$.

A segurança do paciente e a eficiência do procedimento podem ser comprometidas se o enfermeiro perioperatório for inexperiente quanto aos cuidados para pacientes submetidos a procedimentos robóticos. Ao oferecer um programa de treinamento para os enfermeiros envolvidos com a cirurgia robótica, os hospitais proporcionam competência à prática desses profissionais, diminuindo riscos e promovendo resultados positivos para a assistência de enfermagem ${ }^{1}$.

A simulação clínica se tornou uma importante ferramenta na educação da enfermagem como uma alternativa viável para a prática com pacientes. Embora a simulação não possa substituir a prática clínica real, é uma ferramenta útil para a criação de realismo antes do aprendiz realizar o atendimento ao paciente. A simulação incentiva a aprendizagem ativa, estimulando os alunos ${ }^{8}$.

Este artigo possibilita aos coordenadores e educadores de bloco operatório conhecer um programa de treinamento para capacitação de enfermeiros especialistas em cirurgia robótica. Esse modelo pode ser aplicado em outros centros de especialidade, aumentando a expertise desses profissionais. O objetivo deste estudo foi desenvolver um programa de treinamento para enfermeiros residentes em cirurgia robótica.

\section{MÉTODO}

Trata-se de um relato de experiência realizado em um hospital filantrópico privado de grande porte no município de
São Paulo, com 19 salas cirúrgicas e média de 1.200 cirurgias/mês. Nesse hospital foi iniciado, em 2015, um programa de residência de enfermagem em centro cirúrgico e centro de material e esterilização, com dez vagas.

Inserido no calendário pedagógico do programa de residência, a capacitação em cirurgia robótica foi pensada em um formato com carga teórica e prática. Dessa forma, o treinamento foi planejado em conjunto com a empresa responsável pelo sistema Da Vinci: a Intuitive (Figura 1).

O treinamento teórico foi oferecido por meio do site oficial do Da Vinci ${ }^{9}$ e consiste em abranger explicações em videoaulas com exercícios interativos sobre princípios básicos de eletrocirurgia, sistema de funcionamento do Da Vinci, montagem do robô, acoplamento do Da Vinci ao paciente e solução de problemas. Uma avaliação do aprendizado é aplicada ao final do curso - a nota mínima para aprovação é 7,0. Contemplado com a aprovação, o participante recebe um certificado emitido pelo site, o qual deve enviar às tutoras do programa de residência, assim como à representante da empresa a qual comercializa o sistema.

Foi disponibilizado 60 dias para a conclusão do treinamento on-line e acordado que, em caso de reprovação nessa fase, não seria possível progredir para o treinamento prático.

A tutora do programa de residência que acompanha o treinamento prático ficou responsável pela montagem da sala cirúrgica e disposição do sistema junto à engenharia clínica, pelos insumos descartáveis, pela retirada e devolução do instrumental no centro de material e esterilização, pela supervisão da técnica de degermação das mãos, pela paramentação cirúrgica e pela avaliação do treinamento prático.

A capacitação prática foi agendada com antecedência de 60 dias com um representante da Intuitive, para ser realizada em um domingo na sala cirúrgica da robótica no hospital referido; o período para realização dessa fase é de seis horas, e consiste na revisão dos conceitos teóricos aprendidos na primeira fase, na demonstração dos instrumentais específicos para cirurgia robótica e na apresentação do console, sistema de vídeo e robô Da Vinci pelo representante da empresa. A tutora que acompanha o grupo faz a revisão das técnicas de degermação e paramentação cirúrgica.

Durante o processo, as enfermeiras residentes discutem os conceitos aprendidos no grupo e iniciam as atividades práticas com o manuseio dos instrumentais robóticos. Posteriormente, são incentivadas a iniciar individualmente as técnicas de montagem e manuseio do robô, avaliação do funcionamento do sistema e possíveis falhas. Para a montagem 
do robô é requerido a degermação e paramentação cirúrgica para manter o procedimento estéril.

Ao final da capacitação prática, a tutora preenche a avaliação de aquisição das competências esperadas para uma enfermeira especialista em cirurgia robótica. Para isso, foi utilizado um instrumento de avaliação com os conceitos "precisa melhorar" e "satisfatório" (Quadro 1).

\section{RESULTADOS}

Nove enfermeiras residentes foram capacitadas no período de setembro a dezembro de 2015 . O treinamento on-line disponibilizado em setembro foi concluído até novembro, e a prática realizada em dezembro de 2015.

Para o treinamento teórico, as enfermeiras residentes acessaram o site e fizeram um registro com seus dados pessoais; ao final obtiveram um login e uma senha para acesso à área de treinamento.

O treinamento teórico permitiu desde o conhecimento de princípios básicos até a fase final com o dock do robô Da Vinci; durante cada fase, há exercícios para reforço do aprendizado e, ao final, uma avaliação sobre seu conhecimento especifico. No caso de não alcançar a média exigida pelo sistema, todo o treinamento deve ser repetido antes de passar à próxima fase. É permitido retornar ao conteúdo várias vezes, e não há determinação de tempo para execução de cada fase.

O treinamento foi bem avaliado pelas residentes, e, apesar do conteúdo ser em inglês, não tiveram prejuízos quanto ao aprendizado. As enfermeiras residentes conseguiram finalizar o treinamento on-line no tempo proposto.

O treinamento prático foi essencial para visualizar a colocação e operação de equipamentos na sala de cirurgia, entender a posição dos membros da equipe e a organização dos

Identificação da

necessidade de

treinamento

- Optado pelo programa

de treinamento já

existente na empresa,

com sistema de avaliação

do conhecimento cognitivo

\section{com a Intuitive}

- Escolhido uso de simulação no ambiente real, exigido a execução prática de todos os profissionais e somente realizada após aprovação da carga teórica

Figura 1. Programa de capacitação para enfermeiro especialista em cirurgia robótica. 
materiais e instrumentais, praticar o conteúdo aprendido e possibilitar a oportunidade única de manuseio do console, do robô e do sistema de vídeo. A tutora revisitou com as residentes a técnica de degermação das mãos e paramentação cirúrgica e observou a execução do processo de montagem e acoplamento do robô executado individualmente por cada uma das enfermeiras participantes.

As residentes executaram a manipulação dos instrumentos, realizaram a degermação e paramentação como preconizada, realizaram a montagem do robô para a cirurgia, montaram a mesa para o sistema de ótica, identificaram por meio do vídeo a quantidade de usos das pinças e realizaram a desmontagem do sistema. Durante a calibração das óticas e o alinhamento das imagens, o grupo demonstrou algumas dúvidas, que foram esclarecidas. Desse modo, realizaram todas as etapas do processo.

Ao final do treinamento, as residentes foram liberadas para a execução da montagem do robô no cenário intraoperatório. Inicialmente, as participantes devem ser supervisionadas pelo enfermeiro especialista em robótica até que apresentem segurança na montagem do sistema. Em geral, os profissionais requerem três montagens supervisionadas para, posteriormente, executarem a montagem sem monitoramento.

O resultado de nossa experiência foi considerado efetivo para capacitação de enfermeiros em cirurgia robótica.
Os prazos estabelecidos foram suficientes e as competências desejadas foram alcançadas pelos participantes do grupo.

\section{DISCUSSÃO}

O avanço tecnológico e a expansão da cirurgia robótica requerem profissionais capacitados para garantir a segurança do paciente e assertividade do procedimento. Esse avanço tecnológico e a geração dos jovens enfermeiros resultam em mudanças significativas na prática assistencial. Para esse novo perfil de enfermeiro perioperatório, um modelo de treinamento com associação da teoria e prática permite dinamismo ao desenvolvimento desses profissionais, tornando-se efetivo no processo de aprendizagem.

A aprendizagem é um processo ativo e dinâmico, que tem potencial de transformar o aprendiz. Estratégias educacionais eficazes devem ser cooperativas, colaborativas e atraentes para captar e manter a atenção das novas gerações de enfermeiros perioperatórios ${ }^{10}$.

A estratégia aplicada para desenvolver enfermeiras especialistas em cirurgia robótica buscou capacitar enfermeiras residentes em um ambiente simulado da prática profissional, com uso da sala operatória, da disposição dos equipamentos, dos insumos descartáveis e dos instrumentais idênticos ao utilizados no intraoperatório.

Quadro 1. Impresso de avaliação do treinamento prático para cirurgia robótica. São Paulo, 2015.

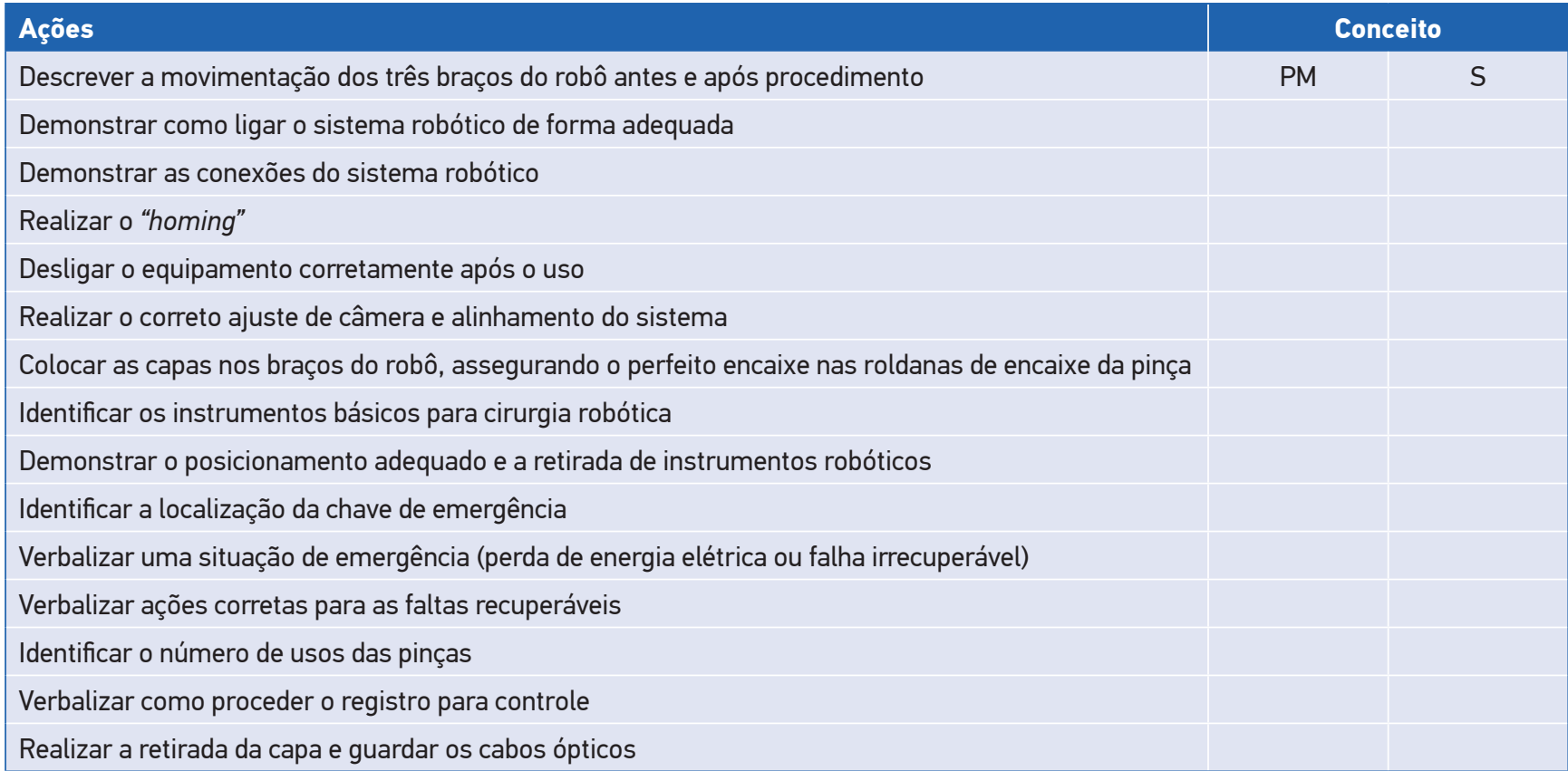

PM: precisa melhorar; S: satisfatório. 
A aplicação de simulação na formação profissional em saúde começou a ganhar destaque com a campanha pela segurança do paciente. A simulação como método de ensino vem ganhando espaço no mundo, tornando-se frequente nos cursos de graduação e pós-graduação em Enfermagem ${ }^{11}$.

Neste estudo, além da simulação na montagem e no acoplamento do sistema robótico, nas habilidades técnicas - como a degermação das mãos e a paramentação cirúrgica — e no manuseio dos instrumentais robóticos, há o reforço desses procedimentos durante o simulado, permitindo a associação do conhecimento prévio com o desenvolvimento da habilidade prática.

Sabe-se que a pós-graduação com modalidade residência caracteriza-se como aprendizado em serviço; possibilita ao residente a prática em saúde; e auxilia esse profissional, em sua maioria recém-formado, a conhecer os processos de trabalho e adquirir segurança profissional em conjunto com a formação crítico-reflexiva ${ }^{12}$. Esse tipo de qualificação eleva a qualidade da assistência e prepara profissionais para o mercado de trabalho ${ }^{13}$.

Esse modelo de treinamento assemelha-se a um estudo americano que desenvolveu um treinamento com carga teórico-prática para enfermeiras em cirurgia robótica. Entretanto, neste estudo, a carga teórica foi desenvolvida pela enfermagem e baseada nas necessidades de sua equipe, sendo a prática inserida posteriormente. O processo levou cinco semanas ${ }^{1}$.
Outro estudo utilizou um treinamento on-line, uma sessão prática de meio período e exercícios de simulação, e separou os participantes em dois grupos - experientes e novatos na prática robótica - obtendo resultados significativos da eficácia desse modelo de treinamento ${ }^{14}$.

Poucos estudos relatam como executam o treinamento de seus enfermeiros para a cirurgia robótica. Em muitas instituições de saúde, o enfermeiro sem conhecimento dessa tecnologia é inserido ao processo de forma gradativa, em acompanhamento de um profissional experiente.

Nesse formato de capacitação, um conhecimento cognitivo prévio associado a uma simulação da prática permite maior segurança ao profissional que inicia a execução da assistência na cirurgia robótica.

\section{CONCLUSÃO}

A capacitação proposta foi satisfatória e atendeu às competências desejadas. O treinamento foi bem avaliado pelas residentes, devido à aquisição de conhecimento teórico e prático. A capacitação de profissionais especialistas em cirurgia robótica é um diferencial na residência de enfermagem perioperatória.

\section{REFERÊNCIAS}

1. Sarmanian JD. Robot-Assisted Thoracic Surgery (RATS): Perioperative Nursing Professional Development Program. AORN J. 2015;102(3):241-53. doi: 10.1016/j.aorn.2015.06.013

2. Brusco JM. Trends in robotic surgery. AORN J. 2012;95(4):1-9. doi: 10.1016/j.aorn.2015.06.013

3. Chan JY, Richmon JD. Transoral robotic surgery (TORS) for benign pharyngeal lesions. Otolaryngol Clin North Am. 2014;47(3):407-13. doi: 10.1016/j.otc.2014.02.003

4. Dutta SR, Passi D, Sharma S, Singh P. Transoral robotic surgery: A contemporary cure for future maxillofacial surgery. J Oral Maxillofac Surg Med Pathol. 2016;28(4):290-303. doi: 10.1016/j.ajoms.2016.03.002

5. Alves AFG, Grassia RCF, Carvalho R. Cirurgia robótica de revascularização miocárdica: em busca de evidência científica no uso da tecnologia do século XXI. Rev SOBECC. 2012;17(3):31-42.

6. Sousa CS, Gonçalves MC, Lima AM, Turrini RNT. Advances in the role of surgical center nurses. J Nurs UFPE. 2013;7(10):6288-93. doi: 10.5205/01012007

7. Thomas CC. Role of the Perioperative Nurse in Robotic Surgery. Perioper Nurs Clin. 2011;6(3):227-34. doi: 10.1016/j.cpen.2011.06.005
8. Neil JA. Simulation in nursing education. Perioper Nurs Clin. 2009;4(2):97-112. doi: 10.1016/j.cpen.2009.02.002

9. da Vinci Surgery Community. da Vinci Surgery Customer Portal. [citado 2016 abr. 20]. Disponível em: www.davincisurgerycommunity.com

10. Smith CE. Developing simulation scenarios for perioperative nursing core competencies and patient safety. Perioper Nurs Clin. 2009;4(2):157-65. doi: 10.1016/j.cpen.2009.01.003

11. Aebersold M, Tschannen D, Bathish M. Innovative simulation strategies in education. Nurs Res Pract. 2012;2012:765212. doi: $10.1155 / 2012 / 765212$

12. Goulart CT, Silva RM, Bolzan MEO, Guido LA. Perfil sociodemográfico e acadêmico dos residentes multiprofissionais de uma universidade pública. Rev Rene. 2012;13(1):178-86.

13. Carmo M. A residência de enfermagem na formação profissional. Ciênc Cuid Saúde. 2013;11(2):223-5.

14. Dulan G, Rege RV, Hogg DC, Gilberg-Fisher KM, Arain NA, Tesfay $\mathrm{ST}$, et al. Developing a comprehensive, proficiency-based training program for robotic surgery. Surgery. 2012;152(3):477-88. doi: 10.1016/j.surg.2012.07.028 